\section{OPEN ACCESS}

Edited by:

Guido laccarino,

University of Naples Federico II, Italy

Reviewed by:

Carlo Gabriele Tocchetti,

University of Naples Federico II, Italy

Claudio Napoli,

Università della Campania Luigi

Vanvitelli, Italy

${ }^{*}$ Correspondence:

Giulia Mingrone

juliamingro@gmail.com;

giulia.mingrone@edu.unito.it

${ }^{\dagger}$ These authors share last authorship

Specialty section:

This article was submitted to

Hypertension,

a section of the journal

Frontiers in Cardiovascular Medicine

Received: 23 December 2020

Accepted: 22 February 2021

Published: 21 April 2021

Citation:

Mingrone G, Astarita A, Airale L, Maffei I, Cesareo M, Crea T, Bruno G, Leone $D$, Avenatti $E$, Catarinella $C$,

Salvini M, Cetani G, Gay F, Bringhen $S$, Veglio $F$, Vallelonga $F$ and Milan A (2021) Effects of Carfilzomib

Therapy on Left Ventricular Function in Multiple Myeloma Patients.

Front. Cardiovasc. Med. 8:645678.

doi: 10.3389/fcvm.2021.645678

\title{
Effects of Carfilzomib Therapy on Left Ventricular Function in Multiple Myeloma Patients
}

\section{Giulia Mingrone ${ }^{1 *}$, Anna Astarita ${ }^{1}$, Lorenzo Airale ${ }^{1}$, Ilaria Maffei ${ }^{1}$, Marco Cesareo ${ }^{1}$, Teresa Crea ${ }^{1}$, Giulia Bruno ${ }^{1}$, Dario Leone ${ }^{1}$, Eleonora Avenatti ${ }^{1}$, Cinzia Catarinella ${ }^{1}$, Marco Salvini ${ }^{2}$, Giusy Cetani ${ }^{2}$, Francesca Gay ${ }^{2}$, Sara Bringhen ${ }^{2}$, Franco Veglio ${ }^{1}$, Fabrizio Vallelonga ${ }^{1 \dagger}$ and Alberto Milan ${ }^{1+}$}

${ }^{1}$ Department of Internal Medicine and Hypertension Division, "Città della Salute e della Scienza" Hospital, University of Turin, Turin, Italy, ${ }^{2}$ Myeloma Unit, Division of Haematology, "Città della Salute e della Scienza" Hospital, University of Turin, Turin, Italy

Background: Carfilzomib improves the prognosis of multiple myeloma (MM) patients but significantly increases cardiovascular toxicity. The timing and effect of Carfilzomib therapy on the left ventricular function is still under investigation. We sought to assess the echocardiographic systo-diastolic changes, including global longitudinal strain (GLS), in patients treated with Carfilzomib and to identify predictors of increased risk of cardiovascular adverse events (CVAEs) during therapy.

Methods: Eighty-eight patients with MM performed a baseline cardiovascular evaluation comprehensive of transthoracic echocardiogram (TTE) before the start of Carfilzomib therapy and after 6 months. All patients were clinically followed up to early identify the occurrence of CVAEs during the whole therapy duration.

Results: After Carfilzomib treatment, mean GLS slightly decreased $(-22.2 \% \pm 2.6$ vs. $-21.3 \% \pm 2.5 ; p<0.001)$. Fifty-eight percent of patients experienced CVAEs during therapy: $71 \%$ of them had uncontrolled hypertension, and $29 \%$ had major CVAEs or CV events not related to arterial hypertension. GLS variation during therapy was not related to an increased risk of CVAEs; however, patients with baseline GLS $\geq-21 \%$ and/or left ventricular ejection fraction (LVEF) $\leq 60 \%$ had a greater risk of major CVAEs (OR $=6.2$, $p=0.004 ; \mathrm{OR}=3.7, p=0.04$, respectively). Carfilzomib led to a higher risk of diastolic dysfunction (5.6 vs. $13.4 \%, p=0.04)$ and to a rise in $E / e^{\prime}$ ratio $(8.9 \pm 2.7$ vs. $9.7 \pm 3.7$; $p=0.006)$.

Conclusion: Carfilzomib leads to early LV function impairment early demonstrated by GLS changes and diastolic dysfunction. Baseline echocardiographic parameters, especially GLS and LVEF, might improve cardiovascular risk stratification before treatment.

Keywords: cardio-oncology, echocardiography, global longitudinal strain, arterial hypertension, cardiovascular organ damage, multiple myeloma 


\section{INTRODUCTION}

Carfilzomib is a second-generation irreversible proteasome inhibitor (PI) approved for the treatment of relapsed or refractory multiple myeloma (RRMM) $(1,2)$. Its efficacy has been established in advanced MM, but the antiproteasome activity is burdened by cardiovascular (CV) adverse effects, such as arterial hypertension, arrhythmia, new-onset or worsening heart failure, dyspnea, coronary heart disease, venous thromboembolism, renal failure, pulmonary hypertension, and cardiac-related sudden death (2-11). Despite these known effects, the exact mechanism and early marker of toxicity of Carfilzomib on cardiac structure and function have not been established. The PI seem to be toxic to cardiomyocytes probably because of the apoptosis caused by accumulation of unfolded, damaged proteins due to proteasome inhibition. In addition, Carfilzomib may induce endothelial dysfunction altering endothelial nitric oxide synthase activity and nitric oxide levels (12-17).

Guidelines and expert position statements (18-20) recommend baseline $\mathrm{CV}$ risk assessment for patients scheduled to receive potentially cardiotoxic therapies, and different approaches for early detection and prevention of $\mathrm{CV}$ diseases have been developing (21-23). The prognostic and predictive role of echocardiographic monitoring in patients undergoing treatment is still under investigation.

Left ventricle ejection fraction (LVEF) has been the most widely used echocardiographic parameter to evaluate cardiotoxicity related to cancer treatments; however, its sensibility in detecting minimal contractility variations is low. Global longitudinal strain (GLS), assessed using 2D speckletracking echocardiography (2D-STE), is a recommended technique for detecting and quantifying early disturbances in LV systolic function $(19,24-26)$. The GLS prognostic value in predicting cardiovascular events in general population and its association with all-cause mortality in patients receiving chemotherapy has already been demonstrated $(27,28)$.

Therefore, the aim of our study is to assess echocardiographic systolic and diastolic changes, including GLS modifications, in patients treated with Carfilzomib, in order to verify their potential predictive value on new incidence of cardiovascular adverse events (CVAEs) during therapy.

\section{METHODS}

\section{Study Design}

From January 2015 to March 2020, 116 MM patients followed by the Myeloma Unit ("Città della Salute e della Scienza," Turin) underwent a baseline CV evaluation before Carfilzomib

\footnotetext{
Abbreviations: MM, multiple myeloma; PI, proteasome inhibitor; RRMM, relapsed or refractory multiple myeloma; LV, left ventricle; LVEF, left ventricle ejection fraction; GLS, global longitudinal strain; 2D-STE, 2D speckle-tracking echocardiography; CV, cardiovascular; CVAE, cardiovascular adverse event; BP, blood pressure; TTE, transthoracic echocardiography; ICC, interclass coefficient correlations; CI, confidence interval; FU, follow-up; LVMi, left ventricular mass indexed to body surface area; RWT, relative wall thickness; LAVi, left atrial volume indexed to body surface area; TDI, tissue Doppler imaging; E/A, transmitral Doppler E wave velocity/transmitral Doppler A wave velocity; E/e ${ }^{\prime}=$ transmitral Doppler $\mathrm{E}$ wave velocity/TDI $\mathrm{e}^{\prime}$ wave velocity.
}

treatment; 88 of them completed the 6 months echocardiographic follow-up (FU) examination and were enrolled. Subsequently, all patients were clinically followed for the duration of chemotherapy to assess the incidence of CVAEs. To be included in the study, patient had to be $>18$ years old and have a diagnosis of MM with clinical indication to Carfilzomib treatment. Patients were excluded in the presence of cardiac amyloidosis or poorquality echocardiographic scans.

The study protocol was approved by the ethic committee of our hospital "A.O.U. Città della Salute e della Scienza" of Turin (Protocol Number 0038655), and each patient signed a written consent form.

Patients underwent a comprehensive cardiovascular evaluation at our EchoLab (Hypertension Unit, University of Turin) before the beginning of Carfilzomib infusions and after 6 months of therapy. The evaluation consisted of clinicalanamnestic assessment, office blood pressure (BP) measurement, electrocardiogram, and transthoracic echocardiography (TTE). Subsequently, all patients were clinically followed up during therapy through periodic review of hematological reports or telephonic interview with a standardized questionnaire aimed at the early identification of CVAEs (Figure 1).

\section{Blood Pressure Measurement}

Office BP measurements were performed according to the current guidelines (29). An automatic sphygmomanometer was used (Omron, M10-IT model). Three BP measurements were performed 1-2 min apart, and the mean value was used for subsequent analysis. Optimal office $\mathrm{BP}$ control was defined as the average $\mathrm{BP}<140 / 90 \mathrm{mmHg}$. In case of uncontrolled $\mathrm{BP}$ values at baseline, antihypertensive treatment was started or optimized.

\section{Echocardiography and 2D Speckle Tracking}

2D TTE and speckle-tracking analysis were performed at baseline (before Carfilzomib therapy initiation) and after 6 months of therapy following the current guidelines (30).

The TTE was performed at rest with the patient lying on the left lateral decubitus position. Standard 2D images were acquired with an iE33 ultrasound machine (Philips Medical System, Andover, MA, USA) equipped with a sector probe (S51 transducer). The images were recorded digitally and analyzed offline by a single operator.

LV diameters and wall thickness were measured in parasternal long-axis view. LV geometry was defined by calculating LV mass (LVM, obtained using the Deveraux formula indexed to body surface area) and relative wall thickness (RWT, obtained dividing the double of the LV inferolateral wall thickness by the LV internal diameter at end diastole). LV diastolic function was defined through the evaluation of early diastolic tissue Doppler (TDI) velocities ( $e^{\prime}$ waves) of septal and lateral mitral annulus, tricuspid regurgitation peak velocity, left atrial volume indexed to body surface area (LAVi), and E/e'ratio. LV diastolic dysfunction can be diagnosed if more than half of the available parameters are abnormal based on the current cutoffs (31). Mitral valve inflow (E and A wave velocity) and deceleration time are traditionally 


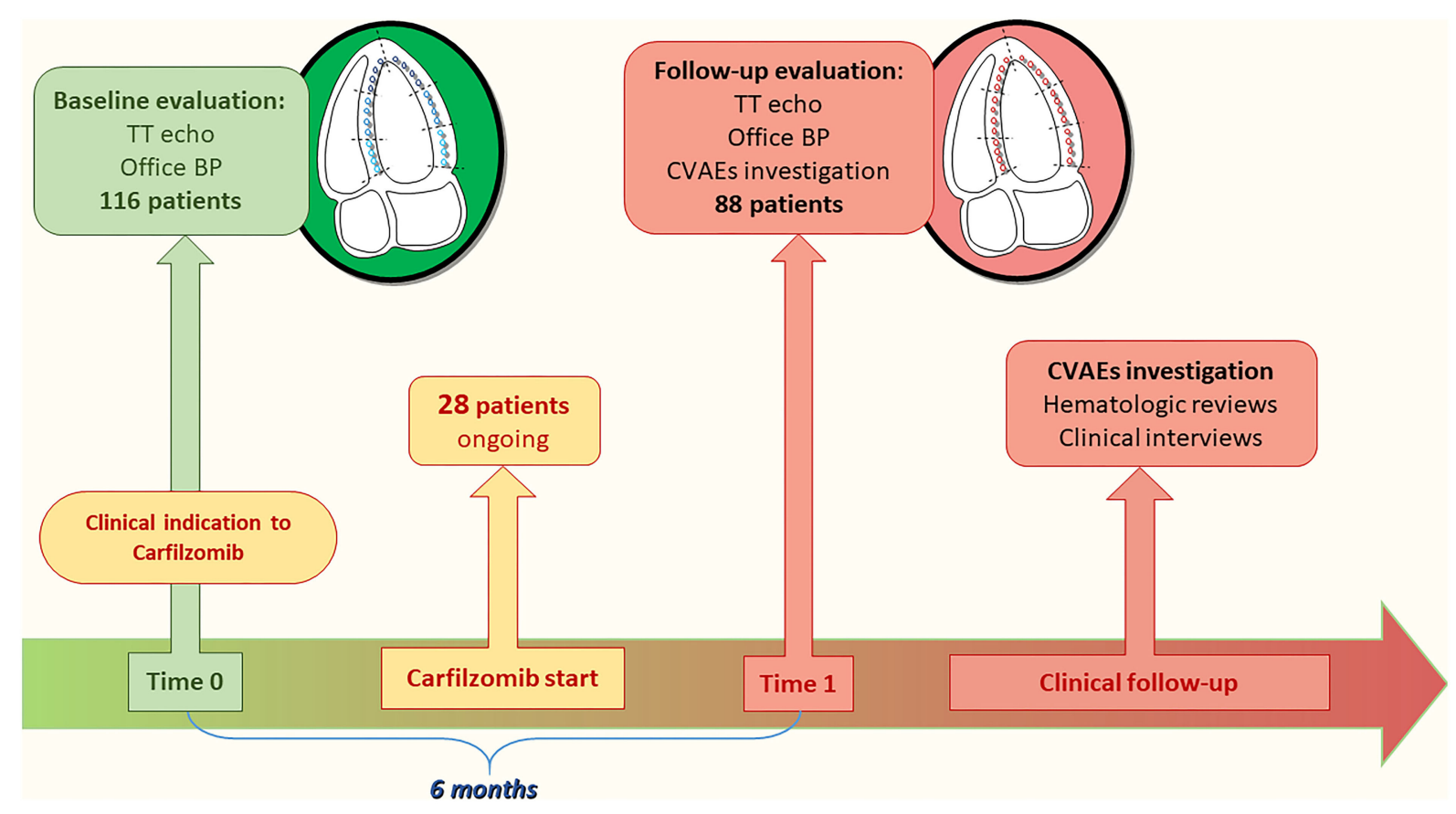

FIGURE 1 | Population and study design. TT, transthoracic; BP, blood pressure; CVAEs, cardiovascular adverse events.

used to identify the filling patterns, according to the current recommendations (31).

STE analysis, including LVEF assessment, was performed with a commercially available software (Automated Cardiac Motion Quantification, QLAB Cardiac Analysis, Philips, Andover, MA, USA). GLS and LVEF were computed offline from standard 2D images of the LV in apical views (4-/2-chambers for LVEF, 4-/3/2-chambers for GLS). The workflow for this analysis requires the endocardium border to be traced semiautomatically after identification of three reference points (apex and mitral annulus), with manual adjustment when needed, following standardized protocols (32). At the end of the GLS analysis, the software output consists of global quantification of strain values (expressed as a $\%$, with more negative values indicating greater deformation), and a "bull's eye" map representing the global and regional LV deformation. LVEF was computed with a semiautomatically Simpson biplane method, based on the 4-/2-chamber volumes. Reproducibility of LVEF and GLS assessments was determined with comparisons of 10 double-blinded measurements, obtained by two expert operators (33).

\section{Cardiovascular Adverse Events Definition}

The incidence of CVAEs was detected both at the 6-month cardiovascular evaluation and later through periodic review of patient hematological reports or phone calls during the whole therapy duration. If severe CVAEs occurred before the planned 6 months FU, the planned repeated TTE examination was anticipated.

CVAEs were assessed and graded according to the Common Terminology Criteria for Adverse Events version 5.0 (34). We divided the CVAEs in arterial-hypertension-related events and non-hypertension related (or "major"). Among the former, we included new onset or worsening arterial hypertension, defined as increased BP values $(\geq 140 / 90 \mathrm{mmHg})$ requiring additional antihypertensive treatments, BP rises occurred just before or immediately after the Carfilzomib infusion (within $30 \mathrm{~min}$ ), uncontrolled hypertension (>180/110 $\mathrm{mmHg}$ ) with related symptoms and without organ damage, hypertensive emergency (symptomatic BP $>180 / 110 \mathrm{mmHg}$ with target acute organ damage caused). Major CVAEs included dyspnea related to Carfilzomib infusions (within 3 days of infusion), arrhythmias (such as atrial fibrillation, atrial bigeminy, ventricular tachycardia, ventricular bigeminy/trigeminy), severe hypotensive events $(\leq 90 / 60 \mathrm{mmHg})$, syncope, cardiac failure, typical chest pain with subsequent negative cardiologic investigations, myocardial infarction, and cardiac arrest.

Based on CVAEs incidence during Carfilzomib treatment, we considered three subgroups: patients who experienced (1) major CVAEs, (2) only arterial-hypertension-related events, and (3) did not experience any adverse event. Patients who experienced both major and arterial-hypertension-related CVAEs were included in group 1 considering the greater clinical implications of major CVAEs.

\section{Statistical Analysis}

Statistical analysis was performed by using SPSS program (IBM SPSS Statistics, Version 22.0.0.0, IBM Corp., Armonk, NY, USA). Quantitative variables were expressed as mean values and standard deviations or median values and interquartile ranges, according to their distribution. Qualitative variables were 
expressed as absolute values and percentages. Paired Student's $t$ test or Wilcoxon test were performed for comparisons before and during/after therapy for quantitative variables, as appropriate, while McNemar test was used for qualitative variables. ANOVA (or non-parametric ANOVA) test was performed to compare quantitative variables between groups; Chi-square test was used for qualitative variables. Logistic regression was utilized to assess the association between baseline echocardiographic parameters and CVAEs risk. A $p<0.05$ was assumed as level of statistical significance for all analysis.

"R A Language And Environment For Statistical Computing" software (v4.0.0 for Mac OSX, R Core Team; Vienna, Austria) was utilized to calculate interclass coefficient correlation (ICC) estimates with their 95\% confident intervals (CIs) (based on a single-rater $k=2$, absolute-agreement, two-way mixed-effects model) and DeLong test used for receiver operating characteristic (ROC) curves comparison.

\section{RESULTS}

A total of 88 patients met the inclusion criteria and represented our study population. Mean age was 65.4 years, with an equal distribution between genders. Half of the patients had history of hypertension, $11 \%$ had diabetes mellitus, and about $16 \%$ dyslipidemia. Median MM duration was 4.6 (2.4-7.1) years (Table 1).

\section{Timing and Dose of Carfilzomib-Based Treatments}

Carfilzomib was administered in association with dexamethasone only (31\% of patients) or immunomodulant drugs plus dexamethasone $(67 \%$ of patients) at a standard dose as International Guideline of MM (1) recommended (Supplementary Table 1). Carfilzomib infusions were continued for a median of $10.4(6.7-18.7)$ months, the median cumulative dose was 3193 (1494.1-5319.3) $\mathrm{mg}$ (at the end of planned therapy or at the time of the study if treatment is ongoing, Supplementary Table 2).

\section{Hemodynamic and Echocardiographic Modifications After Carfilzomib}

Follow-up (FU) cardiovascular evaluations were planned after 6 months of therapy and anticipated, compared to the study plan, whenever a significant event occurred (median, 5.4; interquartile, 4.3-6.4 months). The average cumulative Carfilzomib dose administered until the FU examination was 1413.4 (1140.42105.2) mg. All 88 patients underwent a FU TTE; however, due to poor quality of the apical imaging window, GLS and LVEF analysis was performed on 76 patients $(86.3 \%$ of the population) at baseline and $70(79.5 \%)$ at FU evaluation. Patients in which functional analysis was not possible did not differ in mean age, gender, physical characteristics, and $\mathrm{CV}$ risk factors from the others. ICC results demonstrated an excellent reproducibility for GLS (ICC results and CI $\geq 90 \%$ ) and a good reproducibility for LVEF assessments (ICC and CI $\geq 75 \%$, Supplementary Table 3) (33).
TABLE 1 | Population characteristics at baseline.

\begin{tabular}{|c|c|}
\hline General characteristics & Population, $n=88$ \\
\hline Age, years & $65.4 \pm 8.7$ \\
\hline Male sex, $n(\%)$ & $46(52.3)$ \\
\hline Weight, kg & $71.8 \pm 15$ \\
\hline Height, cm & $161.7 \pm 10.7$ \\
\hline $\mathrm{BSA}, \mathrm{m}^{2}$ & $1.75 \pm 0.2$ \\
\hline $\mathrm{BMI}, \mathrm{kg} / \mathrm{m}^{2}$ & $27.3 \pm 4.4$ \\
\hline \multicolumn{2}{|l|}{ Cardiovascular risk factors } \\
\hline Active smoke, $n(\%)$ & $6(6.8)$ \\
\hline Arterial hypertension, $n(\%)$ & $44(50)$ \\
\hline Obesity, $n(\%)$ & $26(29.5)$ \\
\hline Coronary heart disease, $n(\%)$ & $3(3.4)$ \\
\hline Diabetes mellitus, $n(\%)$ & $10(11.4)$ \\
\hline Chronic renal failure, $n(\%)$ & $9(10.2)$ \\
\hline Dyslipidemia, $n(\%)$ & $14(15.9)$ \\
\hline \multicolumn{2}{|l|}{ Oncological history } \\
\hline MM duration, years & $4.6[2.4-7.1]$ \\
\hline \multicolumn{2}{|l|}{ Previous therapy*: } \\
\hline Anthracyclines, $n(\%)$ & $26(29.5)$ \\
\hline Alkylating agents, $n(\%)$ & $68(77.3)$ \\
\hline Immunomodulating agents, $n(\%)$ & $62(70.5)$ \\
\hline Bortezomib, $n(\%)$ & $76(86.4)$ \\
\hline Auto-transplantation, $n(\%)$ & $63(71.6)$ \\
\hline Relapsed/Refractory MM, $n(\%)$ & $84(95.4)$ \\
\hline
\end{tabular}

${ }^{*}$ Patients were mostly treated with multiple therapies; hence, tot \% will amount to $>100$. $B S A$, body surface area; BMI, body mass index; MM, multiple myeloma.

Office $\mathrm{BP}$ values were reduced at the $\mathrm{FU}$ examination after Carfilzomib, with a greater percentage of BP values $\leq 140 / 90$ mmHg. Forty patients ( $45.5 \%$ of the 88 patients) modified the antihypertensive drug after the baseline visit; 14 (35\%) of these started a new antihypertensive treatment, and 26 (65\%) modified the previous treatment.

Among systolic parameters, only mean GLS (-22.2 \pm 2.6 vs. $-21.3 \pm 2.5, p<0.001)$ showed a statistically significant decrease at FU TTE; morphological parameters remained similar. No statistically significant correlation between GLS variation and cumulative dose of Carfilzomib at FU exam or BP modification was found. The percentage of diastolic dysfunction and $\mathrm{E} / \mathrm{e}^{\prime}$ value increased after therapy, while LAVi and E/A ratio did not show significant changes (Table 2).

\section{Incidence of CVAEs During Carfilzomib Therapy and Baseline Predictors of CVAEs}

Fifty-eight percent of the population experienced CVAEs during a median clinical follow-up of 12.4 (7.9-22.6) months, on average $3.3(0.5-6.3)$ months from Carfilzomib initiation. Of the patients, $52.3 \%$ had hypertension-related CVAEs and about half of them more than one event: specifically, $43.2 \%$ experienced new onset or worsening arterial hypertension, $33 \%$ arterial hypertension just before Carfilzomib infusion and $12.5 \%$ after infusion, $4.5 \%$ uncontrolled hypertension with 
TABLE 2 | Hemodynamic and echocardiographic parameters before Carfilzomib and after 6 months in the whole population.

\begin{tabular}{|c|c|c|c|c|}
\hline \multicolumn{5}{|c|}{ Population, $n=88$} \\
\hline & & Baseline & Follow-up exam & $P$-value \\
\hline Office SBP, mmHg & & $130.3 \pm 18.1$ & $124.6 \pm 15.2$ & 0.003 \\
\hline Office DBP, mmHg & & $76.3 \pm 10.8$ & $72.9 \pm 9.2$ & 0.006 \\
\hline $\mathrm{BP}<140 / 90 \mathrm{mmHg}$ & & $55(63)$ & $69(78.4)$ & 0.013 \\
\hline Antihypertensive ther & & $43(48.9)$ & $61(69.3)$ & $<0.001$ \\
\hline \multicolumn{5}{|l|}{ Echocardiography } \\
\hline \multirow[t]{2}{*}{ LV systolic function } & LVEF, \%* & $60.7 \pm 5.5$ & $59.6 \pm 4.9$ & 0.072 \\
\hline & GLS, \%* & $-22.2 \pm 2.6$ & $-21.3 \pm 2.5$ & $<0.001$ \\
\hline LV morphology & LVMi, $\left(g / m^{2}\right)$ & $90.6 \pm 21.1$ & $90.6 \pm 23.6$ & 0.9 \\
\hline LV diastolic function & Diastolic dysfunction, (\%) & $5(5.6)$ & $11(13.4)$ & 0.04 \\
\hline
\end{tabular}

*Mean values estimated on 70 patients.

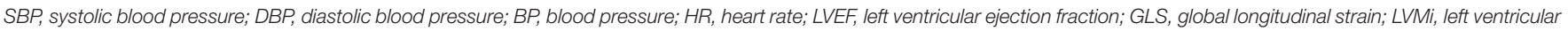

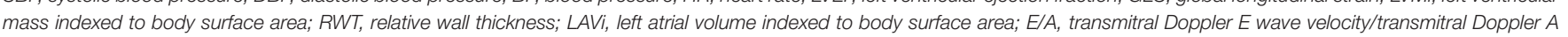
wave velocity; Ele', transmitral Doppler E wave velocity/TDI e' wave velocity.

related symptoms, and no hypertensive emergency reported. Seventeen percent of the patients experienced major CVAEs and about a quarter of them more than one event: $4.5 \%$ had dyspnea, 5.7\% arrythmia (two atrial fibrillation, one atrial bigeminy, one not-sustained ventricular tachycardia, one ventricular bigeminy/trigeminy), $4.5 \%$ severe hypotensive event, $1.1 \%$ heart failure, $3.4 \%$ typical chest pain, $3.4 \%$ patients acute coronary syndrome (one ST elevation and two non-ST elevation myocardial infarction), $1.1 \%$ syncope, and $1.1 \%$ cardiac arrest. Of the patients, $11.3 \%$ experienced both hypertensive and major CVAEs (Supplementary Table 4). There were no differences in mean age, gender, physical characteristics, and CV risk factors between the three groups that were identified based on the occurrence of hypertension-related CVAEs, major CVAEs, or no CVAEs (Table 3). At baseline, patients with no CVAEs had lower systolic and diastolic office mean BP values and a greater proportion of controlled BP. Mean baseline LVEF and GLS values significantly differed between patients with major CVAEs, hypertension-related events, and without events (LVEF, $57.1 \pm$ $4.5 \%$ vs. $60.9 \pm 4.9 \%$ vs. $62.4 \pm 5.6 \%, p=0.007$; GLS, $-20.3 \pm$ $2.4 \%$ vs. $-22.1 \pm 2.2 \%$ vs. $-22.8 \pm 2.7 \%, p=0.008$, respectively), with the greatest difference between patients with major CVAEs and without events. Three patients had a baseline LVEF $<50 \%$ : two of these experienced major CVAEs and one no events. Eleven patients had a GLS $>-19 \%$ at the baseline TTE: six of these experienced major CVAEs, three hypertension-related events, and two no events. Mean baseline GLS and LVEF values predicted the incidence of major CVAEs during Carfilzomib therapy ( $p=$ 0.008 and $\mathrm{OR}=1.407 ; p=0.007$ and $\mathrm{OR}=1.192$, respectively). Through ROC analysis, the best identified baseline GLS value that discriminated an increased risk of major CVAEs was $-21.35 \%$, with a sensitivity of $71.4 \%$ and a specificity of $74.2 \%$ (Figure 2 ). The best baseline LVEF value predicting an increased risk was $60.55 \%$, with a sensitivity of $85.7 \%$ and a specificity of $58.1 \%$ (Figure 2). No statistically significative difference was detected between the area under the ROC curve (AUC) for baseline GLS and LVEF. Considering the rounded baseline values GLS $\geq-21 \%$ and $\mathrm{LVEF} \leq 60 \%$, both predicted an increased risk of major CVAEs $(p=0.004$ and $\mathrm{OR}=6.2 ; p=0.04$ and $\mathrm{OR}=$ 3.7 , respectively).

\section{Hemodynamic and Echocardiographic Variation After Carfilzomib in CVAE-Related Groups}

After 6 months of therapy, we observed a decreasing trend of BP values in all CVAE-related groups (Supplementary Table 5). Subsequently, we analyzed the echocardiographic parameters modifications (Table 4). Patients with major CVAEs showed similar systo-diastolic parameters on TTE before and after therapy. A statistically significant worsening in mean GLS was observed both in patients with hypertensive events and without CVAEs. LVEF was slightly reduced in patients with no adverse events.

Comparisons among groups highlighted a significant difference in terms of GLS variation $(\Delta G L S)$ during Carfilzomib between patients with major CVAEs and patients without events, while LVEF variation ( $\triangle \mathrm{LVEF}$ ) did not differ between groups. No variation in terms of LV morphology (LVMi and RWT) was detected. No statistically significant variation in diastolic parameters was found after Carfilzomib treatment, except for a 
TABLE 3 | General and hemodynamic baseline characteristics according to the type of cardiovascular adverse event.

\begin{tabular}{|c|c|c|c|c|c|}
\hline \multicolumn{2}{|l|}{ Variables } & \multicolumn{4}{|c|}{ Population, $n=88$} \\
\hline Age, years & & $66.1 \pm 5.2$ & $65.8 \pm 9.7$ & $64.6 \pm 8.9$ & 0.8 \\
\hline Male sex, $n(\%)$ & & $7(46.7)$ & $24(66.7)$ & $15(40.5)$ & 0.07 \\
\hline Weight, kg & & $71.1 \pm 15.8$ & $74.9 \pm 14.7$ & $69 \pm 14.7$ & 0.2 \\
\hline $\mathrm{BMI}, \mathrm{kg} / \mathrm{m}^{2}$ & & $27.4 \pm 4.1$ & $27.7 \pm 4.5$ & $27 \pm 4.6$ & 0.8 \\
\hline \multicolumn{6}{|l|}{ CV risk factors } \\
\hline Active smoke, $n(\%)$ & & $1(6.7)$ & $2(5.6)$ & $3(8.1)$ & 0.8 \\
\hline Arterial hypertension & & $8(53.3)$ & $20(55.6)$ & $16(43.2)$ & 0.6 \\
\hline Obesity, $n(\%)$ & & $4(26.7)$ & $9(25)$ & $13(35.1)$ & 0.6 \\
\hline Dyslipidemia, $n(\%)$ & & $1(6.7)$ & $7(19.4)$ & $6(16.2)$ & 0.5 \\
\hline \multicolumn{6}{|c|}{ Office blood pressure } \\
\hline Office SBP, mmHg & & $134.9 \pm 20.7$ & $134.6 \pm 15.3^{\#}$ & $124.2 \pm 18.3^{\#}$ & 0.03 \\
\hline Office DBP, mmHg & & $77.1 \pm 6.8$ & $79.9 \pm 10.1^{\#}$ & $72.2 \pm 12.1^{\#}$ & 0.009 \\
\hline $\mathrm{BP}<140 / 90 \mathrm{mmHc}$ & & $10(66.7)$ & $17(47.2)^{\#}$ & $28(75.7)^{\#}$ & 0.04 \\
\hline $\mathrm{HR}, \mathrm{bpm}$ & & $74.4 \pm 14$ & $76.1 \pm 13$ & $76 \pm 14$ & 0.9 \\
\hline Antihypertensive the & (es), $n(\%)$ & $9(60)$ & $16(44.4)$ & $18(48.6)$ & 0.6 \\
\hline \multicolumn{6}{|c|}{ Baseline echocardiography } \\
\hline \multirow[t]{2}{*}{ LV systolic function } & LVEF, \%* & $57.1 \pm 4.5^{\wedge}$ & $60.9 \pm 4.9$ & $62.4 \pm 5.6^{\wedge}$ & 0.007 \\
\hline & GLS, \% ${ }^{*}$ & $-20.3 \pm 2.4^{\wedge}$ & $-22.1 \pm 2.2$ & $-22.8 \pm 2.7^{\wedge}$ & 0.008 \\
\hline
\end{tabular}

$\# p<0.05$ between groups 2 and 3 .

${ }^{\wedge} p<0.05$ between groups 1 and 3 .

*Mean values estimated on 70 patients (group 1, $n=14$; group 2, $n=30$, group 3, $n=32$ ).

$B S A$, body surface area; BMI, body mass index; MM, multiple myeloma; SBP, systolic blood pressure; DBP, diastolic blood pressure; BP, blood pressure; HR, heart rate; LVEF, left ventricular ejection fraction; GLS, global longitudinal strain; LVMi, left ventricular mass indexed to body surface area; RWT, relative wall thickness; LAVi, left atrial volume indexed to body surface area; E/A, transmitral Doppler E wave velocity/transmitral Doppler A wave velocity; E/e', transmitral Doppler E wave velocity/TDI $e^{\prime}$ wave velocity.

significant increase in $\mathrm{E} / \mathrm{e}^{\prime}$ values in patients with hypertensive events (Table 4).

\section{DISCUSSION}

Carfilzomib seems to cause a mild subclinical impairment of LV systo-diastolic function assessed by TTE. In addition, baseline LVEF and GLS may have a predictive role in identifying patients with an increased risk of CVAEs during treatment.

The efficacy of Carfilzomib in RRMM is well-established, but the antiproteasome activity may have adverse consequences on cardiovascular system. Arterial hypertension is well recognized as one of the most frequent CVAEs $(2-5,35,36)$; moreover, arterial hypertension should be treated before starting Carfilzomib infusions because it is an important predictor of CVAEs (37,
38). In our population, the optimization of antihypertensive treatment led to a better BP control after 6 months. Nevertheless, despite this improved control and specific follow-up, $52.3 \%$ of patients still experienced hypertensive CVAEs, suggesting that optimization of BP values alone could not eliminate the risk of arterial-hypertension-related CVAEs.

At the present time, the predictive and prognostic values of echocardiographic monitoring in patients receiving Carfilzomib are still under investigation, with limited evidences $(4,13,39$, 40). The position statement of the Heart Failure Association, the European Association of Cardiovascular Imaging, and the Cardio-Oncology Council of the European Society of Cardiology (41) suggests an echocardiographic surveillance in medium-/high-risk patients receiving Carfilzomib and strongly recommends prompt echocardiography in the presence of new 


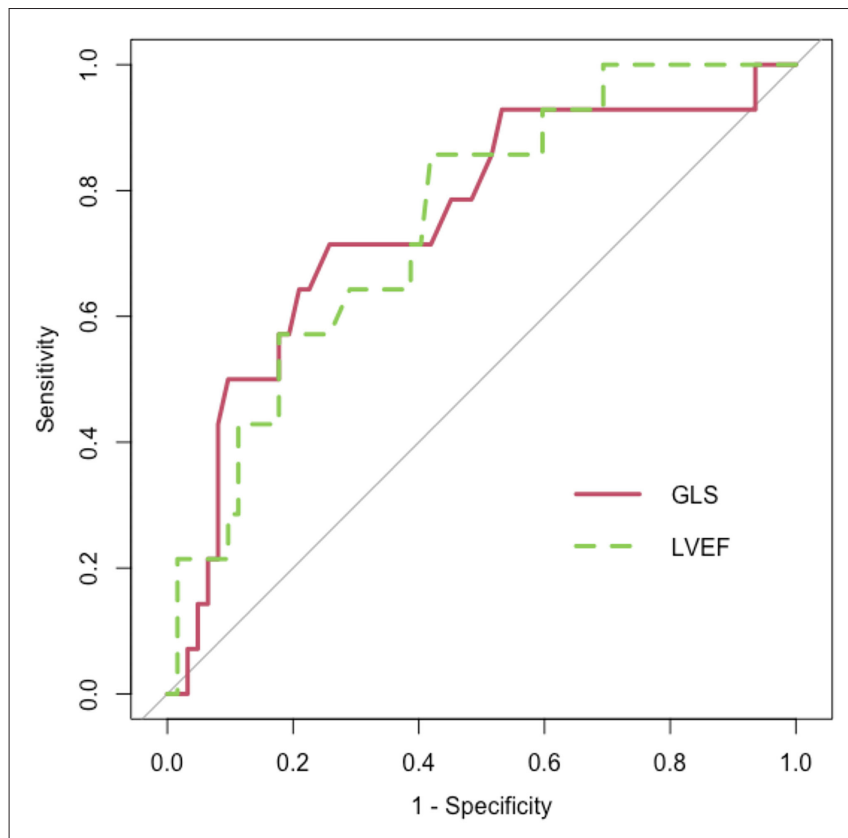

FIGURE 2 | Receiver operating characteristic (ROC) curve (red) for baseline global longitudinal strain (GLS) [area under the curve $(A \cup C)=0.751 ; p=$ $0.004]$ and ROC curve (dashed and green) for baseline left ventricle ejection fraction (LVEF) (AUC $=0.75, p=0.004$ ).

cardiac signs/symptoms. Our study showed that patients treated with Carfilzomib have early (after five to six cycles) but minimal worsening in LV systolic function assessed by GLS $(-22.2 \pm 2.6$ vs. $-21.3 \pm 2.5, p<0.001$ ), in line with previously reported data (42). However, this negative effect on LV systolic function is not predictive of a subsequent clinically relevant cardiac dysfunction. We analyzed echocardiographic changes occurring within few months from the start of therapy (6 months), while CVAEs were recorded over the entire therapy duration (which varies according to the individual hematological indication). It may be possible that cardiac mechanic alterations potentially related to CVAEs were not evident after just 6 months of Carfilzomib treatment, and we could suppose that cardiac alteration that leads to major CVAEs may be evident after more than 6 months of therapy. Moreover, CVAEs incidence may not be entirely explained by cardiac function variations: endothelial dysfunction caused by PI may play an important pathogenetic role (13).

Anyway, worsening of GLS suggests a subclinical damaging effect of Carfilzomib on LV function. Diastolic dysfunction has been explored as a marker of early cardiotoxicity, but the current evidence does not support its role for the prediction of later cardiac dysfunction (43). In our cohort, Carfilzomib showed a detrimental effect on diastolic function: prevalence of diastolic dysfunction in our study population rose from 5.6 to $13.4 \%$, and mean value of $\mathrm{E} / \mathrm{e}^{\prime}$ increased (Table 2). This result is in accordance with previous data that showed a diastolic function change after four cycles of Carfilzomib (44).

In our cohort, $58 \%$ of patients experienced CVAEs: $41 \%$ experienced only hypertensive CVAEs, while $17 \%$ experienced

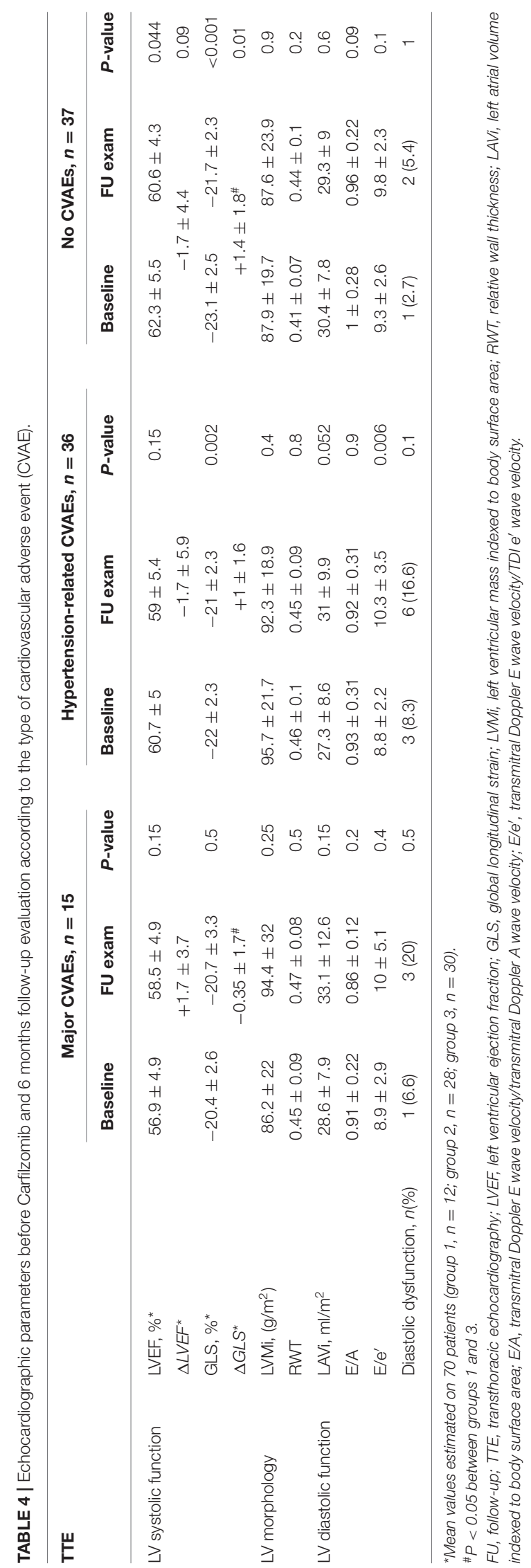


major CVAEs. Patients in the latter group did not show a decrease in functional echocardiographic parameters at the 6 months evaluation, maybe for a limited functional reserve in presence of compromised baseline functional parameters. Hence, in our cohort, a GLS impairment after 6 months of Carfilzomib was not a predictor of CVAEs, while baseline GLS $\geq-21 \%$ and LVEF $\leq 60 \%$ represented predictors of major CVAEs during therapy. We could suppose that Carfilzomib mediates a greater cardiovascular damage, which became clinically evident with the occurrence of CVAEs, in patients with worse functional baseline parameters before beginning a potentially cardiotoxic drug. The echocardiographic baseline functional evaluation could identify the group of patients with an increased risk of major CVAEs if exposed to Carfilzomib treatment, and therefore, it has a relevant prognostic value in MM patients treated with Carfilzomib, in order to identify patients at risk of CV complications.

Our study has some limitations. We have a relatively small cohort, and the GLS analysis with the dedicated software was feasible in $80 \%$ of our patients. Observed incidence of arterial hypertension after Carfilzomib may be limited by the BP optimization at the baseline, but, at the same time, we observed a greater hypertension rate than previously reported in the literature, probably because of our attention as a specialized center (Hypertension Unit). Moreover, FU period was limited by therapy duration; further studies are needed to determine the incidence of long-term CVAEs after Carfilzomib conclusion.

\section{CONCLUSIONS}

In conclusion, our study suggests that Carfilzomib causes an early mild LV systolic function impairment, demonstrated by GLS change after few months of treatment, as well as increases diastolic dysfunction. These functional echocardiographic variations did not directly translate into higher incidence of CVAEs during therapy, reflecting only a subclinical effect on cardiac mechanic. However, baseline echocardiographic parameters may predict the incidence of CVAEs during therapy, and therefore, the echocardiographic baseline assessment plays an essential role in assessing the cardiovascular risk of patients

\section{REFERENCES}

1. Moreau P, San Miguel J, Sonneveld P, Mateos MV, Zamagni E, AvetLoiseau $\mathrm{H}$, et al. Multiple myeloma: ESMO clinical practice guidelines for diagnosis, treatment and follow-up. Ann Oncol. (2017) 28 :iv52iv61. doi: 10.1093/annonc/mdx096

2. Franken B, van de Donk NWCJ, Cloos JC, Zweegman S, Lokhorst HM. A clinical update on the role of carfilzomib in the treatment of relapsed or refractory multiple myeloma. Ther Adv Hematol. (2016) 7:330344. doi: 10.1177/2040620716667275

3. Siegel D, Martin T, Nooka A, Harvey RD, Vij R, Niesvizky R, et al. Integrated safety profile of single-agent carfilzomib: experience from 526 patients enrolled in 4 phase ii clinical studies. Haematologica. (2013) 98:175361. doi: 10.3324/haematol.2013.089334

4. Bringhen S, De Wit E, Dimopoulos, MA. New agents in multiple myeloma: an examination of safety profiles. Clin Lymphoma Myeloma Leuk. (2017) 17:391-407.e5. doi: 10.1016/j.clml.2017.05.003 with clinical indication to Carfilzomib. The presence of one among GLS $\geq-21 \%$ and LVEF $\leq 60 \%$ before therapy initiation may identify patients with an increased risk of experiencing CVAEs during treatment. For these patients, an early initiation of appropriate cardioprotective measures and monitoring might be warranted in order to reduce the incidence of CVAEs and the subsequent therapy withdrawal.

\section{DATA AVAILABILITY STATEMENT}

The original contributions generated for the study are included in the article/Supplementary Material, further inquiries can be directed to the corresponding author/s.

\section{ETHICS STATEMENT}

The studies involving human participants were reviewed and approved by A.O.U. Città della Salute e della Scienza of Turin (Protocol Number 0038655). The patients/participants provided their written informed consent to participate in this study.

\section{AUTHOR CONTRIBUTIONS}

GM wrote the manuscript. AA, AM, and LA contributed to the data analysis. AM, FVa, GB, DL, EA, and FVe revised the manuscript. GC, MS, SB, and FG contributed for the hematologic data and revised the manuscript. All authors contributed to the data collection.

\section{FUNDING}

This research received a research grant from Rete Oncologica Piemonte e Valle d'Aosta (06B/2020/DSM).

\section{SUPPLEMENTARY MATERIAL}

The Supplementary Material for this article can be found online at: https://www.frontiersin.org/articles/10.3389/fcvm. 2021.645678/full\#supplementary-material
5. Bringhen S, Milan A, Ferri C, Wäsch R, Gay F, Larocca A, et al. Cardiovascular adverse events in modern myeloma therapy - incidence and risks. A Review from the European Myeloma Network (EMN) and Italian Society of Arterial Hypertension (SIIA). Haematologica. (2018) 103:142232. doi: 10.3324/haematol.2018.191288

6. Fakhri B, Fiala MA, Shah N, Vij R, Wildes TM. Measuring cardiopulmonary complications of carfilzomib treatment and associated risk factors using the seer-medicare database. Cancer. (2020) 126:808-13. doi: 10.1002/cncr. 32601

7. Bishnoi R, Xie Z, Shah C, Bian J, Murthy HS, Wingard JR, et al. Real-world experience of carfilzomib-associated cardiovascular adverse events: SEER-medicare data set analysis. Cancer Med. (2020) 10:70-8. doi: 10.1002/cam4.3568

8. Rahman MR, Ball S, Paz P, Elmassry M, Vutthikraivit W, Bandyopadhyay $\mathrm{D}$, et al. Heart failure with carfilzomib in patients with multiple myeloma: a meta-analysis of randomized controlled trials. J Card Fail. (2020) S10719164:8-10. doi: 10.1016/j.cardfail.2020.07.006 
9. Mian HS, Fiala MA, Sanchez L, Vij R, Wildes TM. Renal failure among multiple myeloma patients utilizing carfilzomib and associated factors in the "real world." Ann Hematol. (2021) . doi: 10.1007/s00277-021-04420-3. [Epub ahead of print]

10. Waxman AJ, Clasen S, Hwang WT, Garfall A, Vogl DT, Carver $\mathrm{J}$, et al. Carfilzomib-associated cardiovascular adverse events a systematic review and meta-analysis. JAMA Oncol. (2018) 4:e174519. doi: 10.1001/jamaoncol.2017.4519

11. Chari A, Keith Stewart A, Russell SD, Moreau P, Herrmann J, Banchs J, et al. Analysis of carfilzomib cardiovascular safety profile across relapsed and/or refractory multiple myeloma clinical trials. Blood Adv. (2018) 2:163344. doi: 10.1182/bloodadvances. 2017015545

12. Gavazzoni M, Vizzardi E, Gorga E, Bonadei I, Rossi L, Belotti A, et al. Mechanism of cardiovascular toxicity by proteasome inhibitors: new paradigm derived from clinical and pre-clinical evidence. Eur J Pharmacol. (2018). 828:80-8. doi: 10.1016/j.ejphar.2018.03.022

13. Rosenthal A, Luthi J, Belohlavek M, Kortüm KM, Mookadam F, Mayo A, et al. Carfilzomib and the cardiorenal system in myeloma: an endothelial effect? Blood Cancer J. (2016) 6:e384. doi: 10.1038/bcj.2015.112

14. Steiner RE, Manasanch EE. Carfilzomib boosted combination therapy for relapsed multiple myeloma. Onco Targets Ther. (2017) 10:895907. doi: 10.2147/OTT.S102756

15. Hasinoff BB, Patel D, Wu, X. Molecular mechanisms of the cardiotoxicity of the proteasomal-targeted drugs bortezomib and carfilzomib. Cardiovasc Toxicol. (2017) 17:237-50. doi: 10.1007/s12012-016-9378-7

16. Chen-Scarabelli C, Corsetti G, Pasini E, Dioguardi FS, Sahni G, Narula J, et al. Spasmogenic effects of the proteasome inhibitor carfilzomib on coronary resistance, vascular tone and reactivity. EBioMedicine. (2017) 21:206-12. doi: 10.1016/j.ebiom.2017.05.024

17. Raddino R, Gavazzoni M, Pasini F, Gorga E, Vizzardi E, Bonadei I, et al. Cardiovascular toxicity of carfilzomib on vascular tone, vascular reactivity and endothelial function. Vascul Pharmacol. (2015) 75:667. doi: 10.1016/j.vph.2015.11.064

18. Zamorano JL, Lancellotti P, Rodriguez Muñoz D, Aboyans V, Asteggiano R, Galderisi M, et al. (2016). ESC position paper on cancer treatments and cardiovascular toxicity developed under the auspices of the esc committee for practice guidelines. Eur Heart J. (2016) 37:2768-801. doi: 10.1093/eurheartj/ehw211

19. Plana JC, Galderisi M, Barac A, Ewer MS, Ky B, Scherrer-Crosbie M, et al. Expert consensus for multimodality imaging evaluation of adult patients during and after cancer therapy: a report from the american society of echocardiography and the european association of cardiovascular imaging. Eur Heart J Cardiovasc Imaging. (2014) 15:1063-93. doi: 10.1093/ehjci/jeu192

20. Armenian SH, Lacchetti C, Barac A, Carver J, Constine LS, Denduluri N, et al. Prevention and monitoring of cardiac dysfunction in survivors of adult cancers: american society of clinical oncology clinical practice guideline. J Clin Oncol. (2017) 35:893-911. doi: 10.1200/JCO.2016.70.5400

21. Lyon AR, Dent S, Stanway S, Earl H, Brezden-Masley C, Cohen-Solal A, et al. Baseline cardiovascular risk assessment in cancer patients scheduled to receive cardiotoxic cancer therapies: a position statement and new risk assessment tools from the cardio-oncology study group of the heart failure association of the european society. Eur J Heart Fail. (2020) 22:194560. doi: 10.1002/ejhf.1920

22. Zhou Y, Hou Y, Hussain M, Brown SA, Budd T, Tang WHW, et al. Machine learning-based risk assessment for cancer therapy-related cardiac dysfunction in 4300 longitudinal oncology patients. J Am Heart Assoc. (2020) 9:e019628. doi: 10.1161/JAHA.120.019628

23. Benincasa G, Marfella R, Della Mura N, Schiano C, Napoli, Strengths C. Opportunities of network medicine in cardiovascular diseases. Circ J. (2020) 84:144-52. doi: 10.1253/circj.CJ-19-0879

24. Kang Y, Cheng L, Li L, Chen H, Sun M, Wei Z, et al. Early detection of anthracycline-induced cardiotoxicity using two-dimensional speckle tracking echocardiography. Cardiol J. (2013) 20:592-9. doi: 10.5603/CJ.2013.0158

25. Sawaya H, Sebag IA, Plana JC, Januzzi JL, Ky B, Tan TC, et al. Assessment of echocardiography and biomarkers for the extended prediction of cardiotoxicity in patients treated with anthracyclines, taxanes, and trastuzumab. Circ Cardiovasc Imaging. (2012) 5:596-603. doi: 10.1161/CIRCIMAGING.112.973321
26. Potter E, Marwick TH. Assessment of left ventricular function by echocardiography: the case for routinely adding global longitudinal strain to ejection fraction. JACC Cardiovasc Imaging. (2018) 11 (2 Pt 1):26074. doi: 10.1016/j.jcmg.2017.11.017

27. Kuznetsova T, Cauwenberghs N, Knez J, Yang WY, Herbots L, D'Hooge J, et al. Additive prognostic value of left ventricular systolic dysfunction in a population-based cohort. Circ Cardiovasc Imaging. (2016) 9:e004661. doi: 10.1161/CIRCIMAGING.116.004661

28. Rhea IB, Uppuluri S, Sawada S, Schneider BP, Feigenbaum, H. Incremental prognostic value of echocardiographic strain and its association with mortality in cancer patients. J Am Soc Echocardiogr. (2015) 28:66773. doi: 10.1016/j.echo.2015.02.006

29. Williams B, Mancia G, Spiering W, Agabiti Rosei E, Azizi M, Burnier M, et al. ESC/ESH guidelines for the management of arterial hypertension. Eur Heart J. (2018) 39: 3021-104. doi: 10.1093/eurheartj/ehy339

30. Lang RM, Badano LP, Victor MA, Afilalo J, Armstrong A, Ernande L, et al. Recommendations for cardiac chamber quantification by echocardiography in adults: an update from the american society of echocardiography and the european association of cardiovascular imaging. J Am Soc Echocardiogr. (2015) 28:1-39.e14. doi: 10.1016/j.echo.2014.10.003

31. Nagueh SF, Smiseth OA, Appleton CP, Byrd BF, Dokainish H, Edvardsen $\mathrm{T}$, et al. Recommendations for the evaluation of left ventricular diastolic function by echocardiography: an update from the american society of echocardiography and the european association of cardiovascular imaging. $J$ Am Soc Echocardiogr. (2016) 29:277-314. doi: 10.1016/j.echo.2016.01.011

32. Voigt JU, Pedrizzetti G, Lysyansky P, Marwick TH, Houle H, Baumann $\mathrm{R}$, et al. Definitions for a common standard for $2 \mathrm{~d}$ speckle tracking echocardiography: consensus document of the EACVI/ASE/industry task force to standardize deformation imaging. Eur Heart J Cardiovasc Imaging. (2015) 16:1-11. doi: 10.1093/ehjci/jeu184

33. Koo TK, Li MY. A guideline of selecting and reporting intraclass correlation coefficients for reliability research. J Chiropr Med. (2016) 15:15563. doi: $10.1016 / \mathrm{j} . \mathrm{jcm} .2016 .02 .012$

34. Cancer Therapy Evaluation Program (CTEP). Common Terminology Criteria for Adverse Events (CTCAE).v.5.0 [5x7]. Bethesda, MD: CTEP (2017). p. 155. Available online at: https://ctep.cancer.gov/protocoldevelopment/electronic_ applications/ctc.html

35. Milan A, Puglisi E, Ferrari L, Bruno G, Losano I, Veglio F. Arterial hypertension and cancer. Int Cancer J. (2014) 134:226977. doi: $10.1002 / \mathrm{ijc} .28334$

36. Cornell RF, Ky B, Weiss BM, Dahm CN, Gupta DK, Du L, et al. Prospective study of cardiac events during proteasome inhibitor therapy for relapsed multiple myeloma. J Clin Oncol. (2019) 37:1946-55. doi: 10.1200/JCO.19.00231

37. Bruno G, Bringhen S, Maffei I, Iannaccone A, Crea T, Ravera A, et al. Cardiovascular organ damage and blood pressure levels predict adverse events in multiple myeloma patients undergoing carfilzomib therapy. Cancers. (2019) 11:622. doi: 10.3390/cancers 11050622

38. Milan A, Bruno G, Maffei I, Iannaccone A, Ravera A, Schiavone D, Veglio F. Arterial hypertension and multiple myeloma: physiopathology and cardiovascular risk and 'practical' indications in patients receiving carfilzomib. Curr Hypertens Rev. (2018) 15:47-53. doi: 10.2174/1573402114666180611110547

39. Russell SD, Lyon A, Lenihan DJ, Moreau P, Joshua D, Chng J, et al. Serial Echocardiographic assessment of patients (pts) with relapsed multiple myeloma ( $\mathrm{rmm})$ receiving carfilzomib and dexamethasone $(\mathrm{kd})$ vs bortezomib and dexamethasone (vd): a substudy of the phase 3 endeavor trial (NCT01568866). Blood. (2015) 126:4250. doi: 10.1182/blood.v126.23.4250.4250

40. Chari A, Hajje D. Case series discussion of cardiac and vascular events following carfilzomib treatment: possible mechanism, screening, and monitoring. BMC Cancer. (2014) 14:1-9. doi: 10.1186/1471-240714-915

41. Celutkiene J, Pudil R, López-Fernández T, Grapsa J, Nihoyannopoulos $\mathrm{P}$, Bergler-Klein J, et al. The Role of Cardiovascular Imaging in Cancer Patients Receiving Cardiotoxic Therapies: A Position Statement on Behalf of the Heart Failure Association (HFA), the European Association of Cardiovascular Imaging (EACVI) and the Cardio-Oncology Council Of the 
European Society of Cardiology (ESC). Eur J Heart Fail. (2020) 22:150424. doi: 10.1002/ejhf.1957

42. Iannaccone A, Bruno G, Ravera A, Gay F, Salvini M, Bringhen S, et al. Evaluation of cardiovascular toxicity associated with treatments containing proteasome inhibitors in multiple myeloma therapy. High Blood Press Cardiovasc Prev. (2018) 25:209-218. doi: 10.1007/s40292-0180256-1

43. Bringhen S, Milan A, D’Agostino M, Ferri C, Wäsch R, Gay F, et al. Prevention, monitoring and treatment of cardiovascular adverse events in myeloma patients receiving carfilzomib a consensus paper by the european myeloma network and the italian society of arterial hypertension. J Intern Med. (2019) 286:63-74. doi: 10.1111/joim.12882

44. Gavazzoni M, Lombardi CM, Vizzardi E, Gorga E, Sciatti E, Rossi L, et al. Irreversible proteasome inhibition with carfilzomib as first line therapy in patients with newly diagnosed multiple myeloma: early in vivo cardiovascular effects. Eur J Pharmacol. (2018). 838:85-90. doi: 10.1016/j.ejphar.2018.09.014

Conflict of Interest: The authors declare that the research was conducted in the absence of any commercial or financial relationships that could be construed as a potential conflict of interest.

Copyright (c) 2021 Mingrone, Astarita, Airale, Maffei, Cesareo, Crea, Bruno, Leone, Avenatti, Catarinella, Salvini, Cetani, Gay, Bringhen, Veglio, Vallelonga and Milan. This is an open-access article distributed under the terms of the Creative Commons Attribution License (CC BY). The use, distribution or reproduction in other forums is permitted, provided the original author(s) and the copyright owner(s) are credited and that the original publication in this journal is cited, in accordance with accepted academic practice. No use, distribution or reproduction is permitted which does not comply with these terms. 